\title{
Resins for temporary crowns and partial dentures do not differ clinically
}

Luthardt RG, Stössel M, Hinz M, Vollandt R. Clinical performance and periodontal outcome of temporary crowns and fixed partial dentures: a randomized clinical trial. J Prosthet Dent 2000; 83:32-39

Question: Which material provides the best clinical properties for temporary restorations?

Objective To compare the clinical properties of provisional restorations and report the subjective assessment of the patient and the dentists.

Design Prospective randomised controlled trial (RCT) in a university setting using a split-mouth design. The trial was examinerand patient-blinded.

Intervention Thirty patients received 61 temporary restorations for crowns or fixed partial dentures. The temporary restorations were fabricated from four resins using a clear matrix technique. One autopolymerizing resin served as control versus one of three others (dualcure, lightcure and autocure).

Outcome measures Dentists' assessments of different handling characteristics were made according to a modified Californian Dental Association (CDA) scale, ie, good/sufficient/not acceptable. Moreover, intra-oral examination after 2-156 days (average, 37 days) recorded plaque adherence, presence of gingivitis and colour stability, occlusal fit, integrity and retention, and the patient's subjective assessment.

Results The only reported difference was the dentists' assessment of the handling, which ranked the dualcure resin as worse than control. The two autopolymerizing materials had the least reported problems, but the differences were not statistically significant.

Conclusion No significant differences between the temporary resin materials were observed.

Evidence-Based Dentistry (2002) 3, 102. doi:10.1038/ sj.ebd. 6400143

Address for correspondence: Ralph Luthardt, University Hospital Carl Gustav Carus, Fetscherstrasse_74, 01307 Dresden, Germany

\section{Commentary}

An ineffective temporary crown compromises the dental tissues during the time from preparation to placing the final restoration. Quality requirements of a temporary crown include tooth protection, gingival care and tooth support (position of the tooth), but also ease of handling. ${ }^{1}$ Laboratory studies suggest that light-curing resinbased composites are more efficient than conventional self-curing polymethylmethacrylates. Experiences from the field are not univocal, however, and objective clinical data are required.

This paper describes a prospective trial of the effectiveness of temporary restorations made from four materials. The study was designed as a RCT, a brave attempt to give more structure to the available information on temporary crowns. Because of absence of accepted quality guidelines, the researchers developed clinical criteria themselves. Assessments varied from mechanical considerations to dentists' subjective views. Despite these efforts, the paper has shortcomings. Most prominent is the absence of discussion of the choices made during the study. The choices raise interesting points and such a discussion would benefit the inference of the results. For instance, why make a rather small study complex by using both crowns and fixed partial dentures? Although one might argue whether a methacrylate (the material in use the longest) ${ }^{2}$ as a control would have been better, it appeared that the well-chosen splitmouth design did not hold. An uneven distribution was obtained and the paired sampling was not realised. Furthermore, dental students, who lack experience handling these materials, were involved in the clinical work. The moment the perceived ease of handling is assessed, one can doubt its value. It was good to read that a preceding pilot study was performed but, unfortunately, the data from it were not fed into a power-analysis. Finally, the use of well-described statistical tests is suggested in the methods but, without reason, the results are limited to descriptives. This is a pity.

This criticism is not intended to discourage eager researchers. Everyone involved in the field of operative dentistry knows the difficulties of performing clinical studies. These comments are more an incentive to get the best out of the energy expended on a RCT. In this case it could be argued that another study design would serve the aim better, given the sparse clinical data available at this moment.

1. Zarb GE, Bergman B, Clayton JA, Mackay HF (Eds). Prosthodontic Treatment for Partially Edentulous Patients. Saint Louis: Mosby, 1978.

2. Shillingburg HT, Hobo $S$, Whitsett LD, Jacobi R, Brackett SE. Fundamentals of Fixed Prosthodontics. 3rd Edn. Chicago: Quintessence Publishing, 1997.

Cees Kreulen Department of Oral Function and Prosthetic Dentistry, University Medical Center Nijmegen, Nijmegen, The Netherlands 